

\title{
Nanoscale Borromeates
}

Kelly S. Chichak, Andrea J. Peters, Stuart J. Cantrill, and J. Fraser Stoddart*

The California NanoSystems Institute, and Department of Chemistry and Biochemistry

University of California, Los Angeles, 405 Hilgard Avenue, Los Angeles, CA 90095-1569

stoddart@chem.ucla.edu

Received April xx, 2005

\section{SUPPORTING INFORMTION REVISED VERSION}

\section{Correspondence Address}

Dr J F Stoddart

Department of Chemistry and Biochemistry

University of California, Los Angeles

405 Hilgard Avenue, Los Angeles, CA 90095-1569 (USA)

Tel: (+1)-310-206-7078

Fax: (+1)-310-206-1843

E-Mail: stoddart@chem.ucla.edu

* To whom correspondence should be addressed. Tel: (+1)-310-206-7078. Fax: $(+1)-310-206-1843$ 
General Experimental Methods: All solvents were dried prior to use according to literature procedures. Deuterated solvents for NMR spectroscopic analyses were used as received. All reagents and starting materials were purchased and used without further purification. 4,4'-Dinitro2,2'-bipyridyl- $N, N^{\prime}$-dioxide, ${ }^{1}$ 4-bromo-2,6-diformylpyridine (5), ${ }^{2}$ 4-acetoxymethylphenyl boronic acid $^{3}$ (6) and 4-methylthiophenyl boronic acid (7), ${ }^{4}$ were prepared according to known procedures. Thin-layer chromatography (TLC) was carried out either on aluminum sheets coated with silica-gel $60 \mathrm{~F}$ or on aluminum sheets coated with aluminum oxide $60 \mathrm{~F}$. The plates were inspected by UV light. Column chromatography was carried out either by using silica-gel 60 or by using aluminum oxide (neutral, Activity II). Melting points were determined on a melting point apparatus and are uncorrected. All ${ }^{1} \mathrm{H}$ and ${ }^{13} \mathrm{C}$ NMR spectra were recorded at $600 \mathrm{MHz}$ and $150 \mathrm{MHz}$, respectively, or at $500 \mathrm{MHz}$ and $125 \mathrm{MHz}$, respectively. All chemical shifts are quoted in ppm, relative to tetramethylsilane, using the residual solvent peak as a reference standard. Mass spectra were measured with MALDI and ESI ion sources. MALDI Mass spectra were obtained using dihydroxybenzoic acid as the supporting matrix. Electrospray mass spectra were obtained with either $\mathrm{MeOH}$ or $\mathrm{MeCN}$ as the liquid carrier.

(1) Anderson, S.; Constable, E. C.; Seddon, K. R.; Turp, J. E.; Baggot, J. E.; Pilling, M. J. J. Chem. Soc., Dalton Trans. 1985, 2247-2261.

(2) (a) Takalo, H.; Kankare, J. Acta. Chem. Scand., Ser. B 1987, B41, 219-221. (b) Takalo, H.; Pasanen, P.; Kankare, J. Acta. Chem. Scand., Ser. B 1988, B42, 373-377. (c) Takalo, H.; Kankare, J. J. Heterocyclic Chem. 1990, 27, 167-169. (d) Nettekoven, N. Synlett 2001, 12, 1917-1920. (d) Storm, O.; Lüning, U. Eur. J. Org. Chem. 2002, 3680-3685.

(3) Kobayashi, Y.; Tokoro, Y.; Watatani, K. Eur. J. Org. Chem. 2000, 3825-3834.

(4) Brikh, A.; Morin, C. J. Organomet. Chem. 1999, 581, 82-86. 






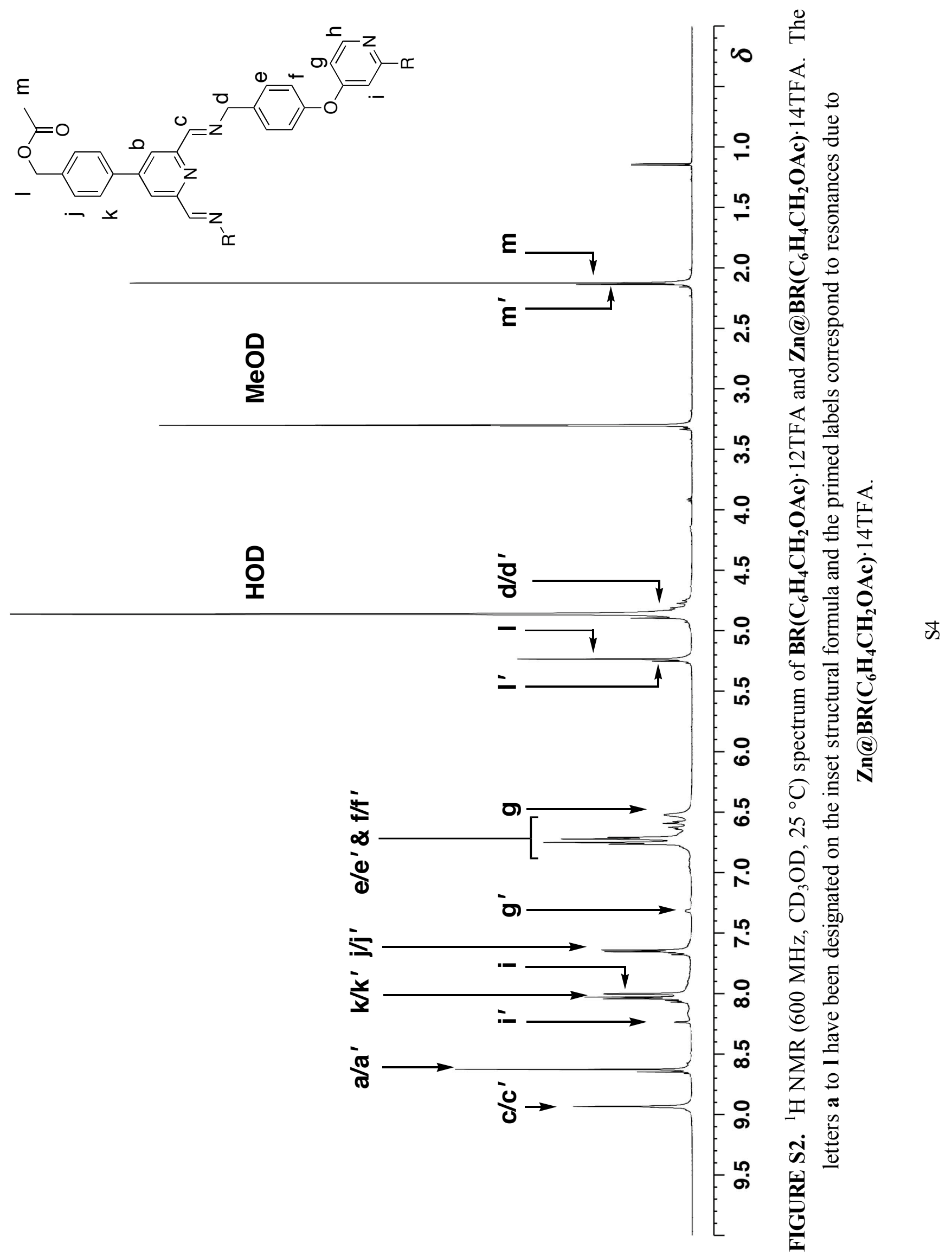